\title{
Perfil socioeconômico-cultural de uma universidade aberta à terceira idade: reflexo da realidade brasileira?
}

Socio-economic and cultural profile in an open university of the third age: a reflection of the brazilian reality?

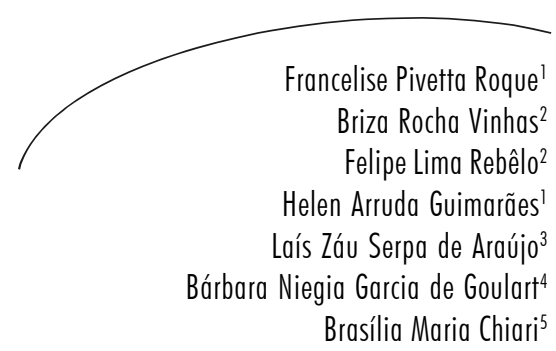

Resumo

Objetivo: comparar os dados socioeconômicos e culturais de alunos de uma Universidade Aberta à Terceira Idade de uma instituição de ensino superior pública alagoana aos dados equivalentes do Estado de Alagoas da população brasileira de mesma faixa etária. Método: estudo transversal comparativo, realizado com os 460 participantes das três primeiras turmas da Universidade Aberta à Terceira Idade (UATI) de uma universidade pública de Alagoas. Compararamse às populações do estado de Alagoas e do Brasil: sexo, idade, escolaridade, composição do domicílio, renda, profissão, estado civil, participação em atividades na comunidade, expectativas em relação à UATI e forma pela qual o aluno havia tomado conhecimento do programa UATI. Resultados: os estudantes da UATI diferem de forma estatisticamente significante da população alagoana e brasileira no que se refere à distribuição de sexo, idade, tipo de domicílio, escolaridade e ocupação $(p \leq 0,05)$. A maioria é casada, exerce atividades religiosas na comunidade, busca crescimento pessoal, além de lazer e distração, e tomou conhecimento da UATI por meio da televisão e amigos. Conclusões: O perfil encontrado não reflete a realidade brasileira, ressaltando a necessidade de que as UATIs, enquanto programas de promoção à saúde, incluam os idosos de classes socioeconômicas e culturais menos favorecidas.

\footnotetext{
Universidade Estadual de Ciências da Saúde de Alagoas (UNCISAL). Maceió, AL, Brasil.

1 Faculdade de Fonoaudiologia. Programa de Extensão Interdisciplinar Pró-Idoso. ${ }^{2}$ Curso de Especialização em Geriatria e Gerontologia. ${ }^{3}$ Departamento de Patologia - Bioética

4 Universidade Federal do Rio Grande do Sul. Porto Alegre, RS, Brasil.

5 Universidade Federal de São Paulo, Departamento de Fonoaudiologia. São Paulo, SP, Brasil.

Estudo desenvolvido em parceria entre o Departamento de Fonoaudiologia da Universidade Federal de São Paulo e o da Universidade Estadual de Ciências da Saúde de Alagoas.
}

Palavras-chave: Idoso. Promoção da saúde. Universidades. Saúde do idoso. Envelhecimento. 
Abstract

Aim: to compare socio-economic and cultural data from participants of a University Open to Seniors of a university in the State of Alagoas to equivalent data from the Brazilian and Alagoas' state population of the same age group. Method: A comparative cross-sectional study conducted with 460 participants from the three first classes of the University for the Third Age (UATI) of a public University in Alagoas. The participants were compared to the population of Alagoas state and Brazil by: sex, age, education, household composition, income and occupation, describing also the marital status, participation in community activities, expectations regarding UATI and the way the student had learned of the UATI program. Results: The students of this UATI show statistically significant difference as compared to the population of Alagoas and Brazil regarding the distribution of sex, age, type of residence, education and occupation $(\mathrm{p} \leq 0.05)$. Most are married, active in the religious community, seeking personal growth, and leisure and distraction in UATI and heard of UATI through television and friends. Conclusions: the profile found does not reflect the Brazilian reality, stressing the need for UATIs, such as programs to promote health, including the elderly of socio-economic and cultural disadvantaged classes.
Key words: Aged. Promotion of health. Universities. Aging health,.Aging.

\section{INTRODUÇÃO}

O processo de envelhecimento populacional no Brasil, cuja população idosa equivale a $10,1 \%,{ }^{1}$ é um dos mais acelerados do mundo. ${ }^{2,3}$ Tendo-se em vista que um país se torna envelhecido quando no mínimo $7 \%$ da sua população é idosa, ${ }^{4}$ é evidente a necessidade de a sociedade brasileira se adaptar a esta nova realidade demográfica, especialmente em estados em que os dados socioeconômicos e culturais são perversos, como Alagoas, onde $8,8 \%$ dos seus três milhões de habitantes são idosos. ${ }^{5}$

Considerando-se que o processo de envelhecimento traz aos indivíduos maior vulnerabilidade às limitações e incapacidades decorrentes das condições adversas do meio físico, social ou afetivo, ${ }^{6,7}$ a Organização Mundial da Saúde (OMS) desenvolveu, em 2005, o conceito de envelhecimento ativo, segundo o qual todas as ações voltadas aos idosos deverão ser fundamentadas no princípio de otimização das oportunidades de saúde, participação e segurança. ${ }^{8}$

Dentro desta perspectiva, a implementação de medidas para ajudar os velhos a se manterem ativos, saudáveis e inseridos socialmente torna-se uma necessidade e, sobretudo, uma responsabilidade por parte das universidades, pelo seu papel de formação educacional, política, de integração e de agente social..$^{3,9}$ Neste contexto, surgem as Universidades Abertas à Terceira Idade (UATIs), com o objetivo de oferecer atividades voltadas às necessidades e à valorização deste público, visando à reinserção social do indivíduo idoso ou com idade próxima a 60 anos. ${ }^{7,10-13}$ Para isto, as UATIs têm como importantes princípios oferecer atividades que tenham relevância social, não com o intuito exclusivo de ocupar o tempo livre deste, e sim preocupando-se com a qualificação e formação de recursos humanos, promoção, cuidado e manutenção da saúde, mediante estratégias de lazer, ensino, cultura e pesquisa. ${ }^{2,13,14}$

A distribuição das UATIs no Brasil é desigual, havendo maior concentração nas regiões mais desenvolvidas (Sul e Sudeste), e, consequentemente, os estudos relacionados a esta parcela nas regiões Norte e Nordeste também se encontram defasados, o que influencia sobremaneira no planejamento e acompanhamento de políticas públicas nessas regiões. ${ }^{12}$ Dessa forma, existe a necessidade de se conhecer melhor 
essa população, a fim de adequar as ações às preferências e necessidades da população idosa, sendo a contribuição deste estudo apresentar resultados em parte da Região Nordeste.

Com base nos aspectos comentados, o presente estudo objetivou comparar os dados socioeconômicos e culturais de alunos de uma Universidade Aberta à Terceira Idade de uma instituição de ensino superior pública alagoana aos dados equivalentes da população de Alagoas e do Brasil, de mesma faixa etária.

\section{MÉTODO}

Este é um estudo comparativo entre o perfil socioeconômico e cultural dos 460 participantes da única, à época, Universidade Aberta à Terceira Idade (UATI) pública de Alagoas, ligada a uma instituição de ensino superior estadual, e os dados da população de Alagoas e do Brasil, em relação a sexo, idade, escolaridade, composição do domicílio, renda e ocupação. Além disso, foram descritos o estado civil, a participação em atividades na comunidade, as expectativas em relação à UATI e a forma de divulgação pela qual o aluno se utilizou para tomar conhecimento da existência desta UATI.

Os sujeitos incluídos nesta pesquisa corresponderam aos alunos das três primeiras turmas de um projeto de extensão que integra um programa de extensão interdisciplinar na área do envelhecimento, tendo como públicoalvo pessoas de 50 anos ou mais, em sua maioria independente e ativa. As atividades foram iniciadas no segundo semestre de 2006, tendo sido as duas primeiras turmas de duração semestral, e da terceira em diante, anual. Foram oferecidas atividades diversas, como o curso denominado Envelhecimento Ativo, no qual foram tratados assuntos sobre saúde, cultura e sociedade na frequência de três vezes por semana, e oficinas de temas variados, incluindo inglês, espanhol, artes, informática, equilíbrio e postura, dança, origami, biodança e etiqueta social.
Os dados dos alunos da UATI foram adquiridos por consulta aos questionários, nos quais havia questões investigativas a respeito das variáveis analisadas neste trabalho, preenchidos por estes no ato da matrícula. Os dados nacionais e de Alagoas foram obtidos nos principais bancos de dados brasileiros: o Instituto Brasileiro de Geografia e Estatística (IBGE), ${ }^{1}$ a síntese dos indicadores sociais relativas a idosos em 2007, e a Pesquisa "Idosos no Brasil-2007", realizada pelo Serviço Social do Comércio (SESC). ${ }^{15} \mathrm{~A}$ análise das idades foi realizada seguindo-se as proporções das faixas etárias sugeridas pelo IBGE (2007), assim como nas variáveis sexo, escolaridade, renda, composição do domicílio e ocupação. O estado civil foi descrito traçando-se comentários comparativos com os dados advindos do SESC.

Os dados foram tabulados e processados pelo aplicativo para microcomputador Statistical Packeage for Social Sciences (SPSS) versão 16.0. Aplicou-se o teste qui-quadrado para comparação das variáveis gênero, faixa etária, renda e domicilio entre a UNCISATI (Universidade Aberta à Terceira Idade da Universidade Estadual de Ciências da Saúde de Alagoas) e os dados disponiblizados na literatura. Utilizou-se o teste exato de Fisher para as variáveis escolaridade e ocupação, quando $20 \%$ das frequências esperadas ou teóricas foram inferiores a 5 ou quando qualquer uma delas foi inferior a 1. Os valores foram considerados significativos para $\mathrm{p}$ menor que $0,05(\mathrm{p}<0,05)$. O valor do erro beta admitido foi de 0,1 .

O projeto desta pesquisa foi aprovado pelo Comitê de Ética em Pesquisa da UNCISAL, protocolo número 824 , tendo-se respeitado todos os preceitos éticos, conforme a Declaração de Helsinque e a Resolução no 196/96 (BRASIL, 1996), garantidos aos sujeitos da pesquisa, entre outros, os direitos ao sigilo das informações e à privacidade.

\section{RESULTADOS}

Conforme pode ser visualizado na tabela 1 , no que se refere à distribuição da frequência por sexo da população estudada, verificou-se que a 
maioria dos alunos da UATI é de mulheres, assim como acontece em Alagoas e no Brasil. No entanto, a distribuição da população por sexo na UATI é diferente, estatisticamente significante, da encontrada em Alagoas e no Brasil $(\mathrm{p}<0,001)$.

Tabela 1 - Distribuição da frequência (\%) de sexo da população da UATI, de Alagoas e do Brasil.

\begin{tabular}{lccc}
\hline & Mulheres & Homens & Total \\
\hline Nacional & 55,93 & 44,07 & 100 \\
Alagoas & 55,59 & 44,41 & 100 \\
UATI** & 93,3 & 6,7 & 100 \\
\hline
\end{tabular}

* Fonte: IBGE, Uma análise das condições de vida da população brasileira, 2007; **(p < 0,001).

A comparação dos dados da UATI aos de Alagoas referentes à distribuição da frequência da idade demonstrou diferença estatisticamente significante $(\mathrm{p}=0,024)$, assim como a comparação aos dados brasileiros, cuja diferença foi ainda maior $(\mathrm{p}<0,001)$ (tabela 2).

Tabela 2 - distribuição da frequência (\%) da idade da população da UATI, de Alagoas e do Brasil.

\begin{tabular}{lcccccccc}
\hline & 50 A 59 & 60 A 64 & 65 A 69 & 70 A 74 & 75 A 79 & $>80$ & Total \\
\hline Nacional & 25,02 & 23,01 & 18,56 & 14,11 & 9,65 & 9,65 & 100 \\
Alagoas & 42,14 & 16,35 & 13,84 & 10,69 & 6,92 & 10,06 & 100 \\
UATI & 48,10 & 21,30 & 18,50 & 5,80 & 4,70 & 1,70 & 100 \\
\hline
\end{tabular}

* Fonte: IBGE, Uma análise das condições de vida da população brasileira, 2007.

Em relação à análise da renda da UATI, também tanto quando comparada à população de Alagoas se verificou diferença estatisticamente significante, $\quad(p<0,001)$, quanto à do Brasil $(p<0,001)$.

Tabela 3 - Distribuição da frequência (\%) da renda da população da UATI, de Alagoas e do Brasil.

\begin{tabular}{lccccc}
\hline & aposentados & pensionistas & $\begin{array}{c}\text { aposentados e } \\
\text { pensionistas }\end{array}$ & $\begin{array}{c}\text { outras - individual e } \\
\text { familiar }\end{array}$ & Total \\
\hline Nacional & 57,1 & 12,1 & 7,4 & 23,4 & 100 \\
Alagoas & 65,4 & 6,1 & 7,3 & 21,2 & 100 \\
UATI** & 43,6 & 5,8 & 5,2 & 45,4 & 100 \\
\hline
\end{tabular}

* Fonte: IBGE, Uma análise das condições de vida da população brasileira, 2007; ** p < 0,001. 
Quando comparada a distribuição da frequência de escolaridade da UATI aos dados de Alagoas e do Brasil, apresentados na tabela 4 , houve diferença estatisticamente significante $(p<0,001)$. Verifica-se que a grande maioria da população da UATI (78.7\%) estudou mais de nove anos, dado oposto ao da população de Alagoas e do Brasil, em que as pessoas com tal nível de escolaridade correspondem à minoria.

Tabela 4 - Distribuição da frequência (\%) da escolaridade da população da UATI, de Alagoas e do Brasil.

\begin{tabular}{lccccc}
\hline & $<1$ ano & 1 a 3 anos & 4 a 8 anos & $>9$ anos & Total \\
\hline Nacional & 33,5 & 20,6 & 31,5 & 14,4 & 100 \\
Alagoas & 60,6 & 14,4 & 16,7 & 8,3 & 100 \\
UATI** & 14,3 & 0,3 & 6,7 & 78,7 & 100 \\
\hline
\end{tabular}

* Fonte: IBGE, Uma análise das condições de vida da população brasileira, 2007; ** p < 0,001.

No que se diz respeito à composição do domicílio, apresentada na tabela 5 , verificou-se diferença estatisticamente significante quando a
UATI foi comparada aos dados de Alagoas $(\mathrm{p}=0,033)$ e, de forma mais expressiva, à do Brasil $(\mathrm{p}<0,001)$.

Tabela 5 - Distribuição da Frequência (\%) da composição do domicílio da população da UATI, de Alagoas e do Brasil.

\begin{tabular}{lcccccc}
\hline & sozinho & $\begin{array}{c}\text { casal sem } \\
\text { filhos }\end{array}$ & $\begin{array}{c}\text { sem filhos e com } \\
\text { outros }\end{array}$ & $\begin{array}{c}\text { com filhos e } \\
\text { outros * }\end{array}$ & Outros * & Total \\
\hline Nacional & 13,2 & 22,3 & 10,7 & 44,4 & 9,4 & 100 \\
Alagoas & 10,9 & 16,3 & 13,2 & 51,6 & 8 & 100 \\
UATI & 8,9 & 10,9 & 12,5 & 65,8 & 1,9 & 100 \\
\hline
\end{tabular}

* Fonte: IBGE, Uma análise das condições de vida da população brasileira, 2007.

No que se refere à posição na ocupação de trabalho principal da população estudada, a UATI se mostrou diferente da população alagoana e brasileira $(\mathrm{p}<0,001)$. A maioria dos alunos $(60,4 \%)$ possuía algum tipo de vínculo empregatício. Nenhum indivíduo deste estudo foi classificado como trabalhador doméstico ou trabalhador somente para consumo próprio. Em comparação com os dados nacionais e de Alagoas, destacou-se significante disparidade em relação ao tipo de ocupação atual, visto que os dados nacionais e estaduais demonstram que a maioria dos idosos trabalha por conta própria, ao contrário dos dados encontrados na UATI, cuja maioria se classifica como possuir algum tipo de vínculo empregatício, como descrito na tabela 6. 
Tabela 6 - Distribuição da Frequência da posição na ocupação no trabalho principal da população da UATI, de Alagoas e do Brasil.

\begin{tabular}{lccc}
\hline & Nacional & Alagoas & UATI $^{* *}$ \\
\hline Empregado & 16,8 & 11,5 & 60,4 \\
Militar e Estatutário & 4,5 & 4,1 & 15 \\
Conta própria & 39,9 & 44,5 & 15,1 \\
Consumo próprio & 22 & 24 & 0 \\
Sem remuneração & 5,2 & 6,4 & 9,7 \\
Trabalhador Doméstico & 4,7 & 2,6 & 0 \\
Total & 100 & 100 & 100 \\
\hline
\end{tabular}

* Fonte: IBGE, Uma análise das condições de vida da população brasileira, 2007; ** $\mathrm{p}<0,001$.

No que concerne ao estado civil da população estudada, visualizada na tabela 7 , a maioria (41,96\%) dos alunos da UATI é casada, à semelhança do que se observa na pesquisa nacional realizada pelo SESC (52\%), seguida dos solteiros e viúvos e, em menor proporção, os divorciados. Quando comparados aos dados do SESC, percebem-se diferenças percentuais, pois o percentual de solteiros nacional (6\%) é bem menor do que a taxa encontrada $(30,87 \%)$ neste estudo, e em relação ao número de viúvos, o contrário aconteceu, onde o índice menor se deu na UATI (17,60\%) estudada, enquanto que no SESC foi de $34 \%$.

Analisando-se estes dados segundo o sexo, observa-se que, dentre os homens, não havia viúvos no público da UATI As mulheres foram responsáveis por $17,60 \%$ do total de viúvos. $\mathrm{Na}$ pesquisa do SESC, a maioria dos viúvos foi formada pelas mulheres (48\%), enquanto que os homens corresponderam à minoria (14\%).

Tabela 7 - Distribuição numérica e percentual dos dados relacionados ao estado civil da população da UATI.

\begin{tabular}{lcccccccc}
\hline Estado civil & \multicolumn{3}{c}{ Brasi $^{*}$} & \multicolumn{3}{c}{ UATI } & & \multicolumn{2}{c}{ Total (\%) } \\
& Fem. & Masc. & Fem. & \multicolumn{2}{c}{ Masc. } \\
\hline & $\%$ & $\%$ & $\mathrm{~N}$ & $\%$ & $\mathrm{~N}$ & $\%$ & Nacional & UATI \\
Casado & 37 & 73 & 176 & 38,26 & 17 & 3,70 & 52 & 41,96 \\
Solteiro & $\mathrm{nc}$ & $\mathrm{nc}$ & 134 & 29,13 & 8 & 1,74 & 6 & 30,87 \\
Viúvo & 48 & 14 & 81 & 17,60 & 0 & 0 & 34 & 17,60 \\
Divorciado & $\mathrm{nc}$ & $\mathrm{nc}$ & 38 & 8,26 & 4 & 0,87 & $\mathrm{nc}$ & 9,13 \\
Outro & $\mathrm{nc}$ & $\mathrm{nc}$ & 1 & 0,22 & 1 & 0,22 & $\mathrm{nc}$ & 0,44 \\
\hline
\end{tabular}

nc - não consta na pesquisa do SESC onde os dados foram coletados.

* Fonte: SESC, Idosos no Brasil: vivências, desafios e expectativas na terceira idade, 2007. 
Em relação à participação dos alunos da UATI em outras atividades da comunidade em que se encontram inseridos, a maioria $(46,1 \%)$ participava de atividades religiosas, sendo $43,7 \%$ deles, mulheres; $11,3 \%$ dos indivíduos realizavam algum tipo de atividade física. Participavam de atividades filantrópicas $7,1 \%$ dos indivíduos, e frequentavam grupos de convivência $3 \%$ dos integrantes deste estudo. As atividades exercidas na comunidade foram qualificadas como uma atividade profissional (seu trabalho) por 29 (6,3\%) dos alunos. Dezesseis (3,5\%) dos participantes do estudo não foram classificados em nenhuma das categorias acima citadas, mas desenvolviam algum outro tipo de atividade que não fora contemplada nas opções oferecidas. Dos participantes, 7,2\% não exerciam atividades em suas comunidades.

Sobre suas expectativas em relação à UATI, $45,2 \%$ dos alunos almejavam por crescimento pessoal, e 32,4\% buscavam a UATI objetivando lazer e distração. Visavam à prática de atividades culturais $8,7 \%$ dos participantes, e à educação, $3,7 \%$ dos alunos. Matricularam-se na UATI com o objetivo de alcançar conhecimentos em saúde $5 \%$ dos alunos, e 2,2\% dos alunos buscavam conhecimentos sobre seus direitos civis. Selecionaram outro motivo não explicitado nas opções do questionário 1,9\% dos alunos.

Em relação à forma como tomaram conhecimento da UATI, a televisão mostrou-se o meio ao qual mais se recorreu para divulgação, utilizada por $37,7 \%$ dos participantes, sendo $33,7 \%$ deles, mulheres. A segunda forma mais comum de divulgação foi a realizada informalmente entre os participantes ou exparticipantes, responsável por $25 \%$ dos casos, dos quais $24,6 \%$ eram mulheres. A divulgação realizada por funcionários da instituição correspondeu a $10,6 \%$ dos casos, $10,4 \%$ deles, mulheres. Tomaram conhecimento da UATI por meio de jornais impressos $10 \%$ dos alunos sendo 9,1\% mulheres, $2 \%$ do público da UATI, souberam desta através de informações em centros de convivência, sendo todas mulheres. Registraram que tomaram conhecimento da UATI via rádio $1,1 \%$, onde $0,7 \%$ eram mulheres, e de outra forma não contemplada nas opções oferecidas na ficha de inscrição $10,2 \%$ dos participantes deste estudo, sendo $9,8 \%$ mulheres.

\section{DISCUSSÃO}

A maior concentração de mulheres em relação a homens verificada na população da Universidade Aberta da Terceira Idade, em foco neste estudo (tabela 1), quando comparada às proporções na população de Alagoas e do Brasil, aponta o resultado que em geral é encontrado nos trabalhos de perfil em UATIs, demonstrando que a participação masculina dificilmente passa de $20 \%$. ${ }^{211}$ Além de o processo de envelhecimento se apresentar com predominância feminina e a expectativa de vida da mulher ser mais alta que do homem, ${ }^{16,17}$ esta conjuntura pode também se justificar pelo pouco interesse provavelmente despertado em homens por este tipo de atividade com caráter cultural, educacional, lúdico e social, sobretudo num estado culturalmente tradicional, em que existe preconceito acerca da participação destas atividades, anteriormente caracterizadas como femininas.

De acordo com o exposto na tabela 2, a população estudada apresenta predomínio de indivíduos ainda não considerados idosos (até 59 anos) e idosos jovens (até 69 anos). ${ }^{3}$ Isso se explica pelo fato de o envelhecimento da população brasileira ser um processo recente, embora seja um dos mais acelerados do mundo, determinando essa concentração dos idosos em faixas etárias mais jovens. No entanto, esta concentração UATI é maior do que a encontrada na população, o que condiz com relatos de outras experiências de UATIs, ${ }^{2,10}$ nos quais os participantes são descritos como tendo perfil de saúde satisfatório, em sua maioria independentes, com boa cognição e desejo de estabelecer e manter contato social.

As diferenças de renda da população estudada em relação às populações alagoana e brasileira (tabela 3) demonstram que os participantes da UATI ainda se encontram economicamente ativos, sem terem se aposentado, possivelmente pelo fato de predominarem adultos maduros e idosos jovens. 
Além disso, devido ao fato de a Região Nordeste apresentar um baixo nível de poder aquisitivo e baixos valores de aposentadoria e/ou pensão, muitos idosos se mantêm ativos ou dependendo dos familiares na tentativa de manter o padrão de vida.

Em relação à composição do domicílio, conforme se visualiza na tabela 5, a maior parte dos alunos mora com filhos e outros, constituindo, desta forma, um grande número de domicílios multigeracionais, de modo semelhante ao que ocorre em Alagoas e no Brasil, o que pode favorecer o fortalecimento das relações familiares, proporcionando aos idosos e aos familiares mais jovens a convivência intergeracional, além de permitir que esses indivíduos atuem como provedores de parte da renda familiar. ${ }^{16,19,20}$ Apesar destes aspectos positivos, a inserção familiar do idoso neste ambiente pode, contrariamente, provocar conflitos, afetando diretamente a qualidade de vida do idoso. ${ }^{21}$

Na UATI estudada, a distribuição dos idosos morando sozinhos ou com cônjuges, sem filhos, difere não só da população de Alagoas e do Brasil, como também do que foi observado em outras pesquisas em UATIs (Universidade do Estado do Rio de Janeiro-UERJ e Universidade Federal de Pernambuco-UFPE), ${ }^{2}$ em que se verificou um número de idosos morando sozinho superior aos valores nacionais, enquanto que no presente estudo os números encontrados foram menores que os dados de Alagoas e nacional. Isso pode ter ocorrido pelo fato de a população deste estudo apresentar maior dependência econômica familiar por ser Alagoas o estado que apresenta maior disparidade e desigualdade social, o que não possibilita ao idoso manter-se em uma casa sozinho, nem seus familiares se manterem em outra casa por precisarem, da aposentadoria de pais e avós para sobreviver. Este dado, no entanto, configura-se como protetor para os idosos desta UATI: mesmo que morar sozinho seja uma escolha, este dado é considerado como um fator de risco aumentado para a saúde deste indivíduo, ao se pensar nas chances de suscitar um processo de isolamento (pela dificuldade de contato com outros familiares), perda de autonomia (dentre outros fatores, pelo aumento do risco de queda) e inadequado apoio familiar, o que se torna minimizado nesta população da UATI, pelo pequeno percentual evidenciado de moradia unipessoal. ${ }^{10,21}$

No que se refere à ocupação dos sujeitos, demonstrada na tabela 6 , a maioria possuía algum vínculo empregatício, resultado que se contrapõe aos dados socioeconômicos nacionais e do Estado de Alagoas, indicando que pequena parcela da população idosa estava empregada. Tal divergência pode ser entendida à luz da diferença do nível de escolaridade entre as populações comparadas neste estudo, ou seja, o melhor nível de escolaridade dos alunos da UATI, se comparada a do grupo da população em geral. É uma circunstância que pode ter conferido ao grupo pesquisado possibilidades de inserção ao mercado de trabalho, para as quais a maioria da população idosa brasileira está aquém. Os dados também diferem dos encontrados em UATI pernambucana, na qual a maioria das mulheres, de níveis socioeconômicos e culturais semelhantes aos da UATI alagoana, relatou ter ocupação anterior de dona de casa. ${ }^{2} \mathrm{O}$ autor desta descrição em Pernambuco atribui seus resultados ao fato de estas mulheres terem pertencido a uma classe e uma época onde o papel principal da mulher era o cuidado da casa e da família. Assim, pode-se atribuir a diferença de ocupações a uma particularidade da população do presente estudo.

Pôde-se verificar ainda que os alunos da UATI pesquisada divergem da maioria da população idosa brasileira e de Alagoas, bem como de outros estudos ${ }^{10}$ no que diz respeito à pequena porcentagem de sujeitos que referem trabalho por conta própria, possivelmente explicada pela escolaridade. A proporção de idosos com escolaridade inferior a um ano em Alagoas, demonstrado na tabela 4, aproxima-se do dobro da proporção de idosos da população em geral, refletindo a situação de precariedade a que aqueles idosos estão submetidos, tendo-se em vista que o baixo nível educacional figura dentre os fatores que, associados entre si, comprometem o processo de senectude, trazendo grandes danos para sua qualidade de vida, sendo eles a idade avançada e o sexo feminino. ${ }^{1,9,15,20} \mathrm{~A}$ isto se sobrepõe of fato de 
que o acesso à educação na época em que nossos idosos eram crianças era mais difícil, sobretudo para as mulheres.

Na mesma tabela, verifica-se que a população que chega à UATI não reflete, em termos de escolaridade, a realidade geral, pois $78,7 \%$ têm mais que nove anos de estudo, e apenas 14,3\% apresentam menos que um ano de estudo, ou seja, trata-se de idosos em melhor condição socioeconômica e cultural, e menor situação de vulnerabilidade do que a maioria da população no Estado de Alagoas. Uma das hipóteses para explicar esta situação é a de que, devido à baixa renda da maioria da população alagoana, os idosos necessitam trabalhar e, portanto, não apresentam o tempo livre que se espera nesta etapa da vida, não sendo a escolaridade em si o motivo direto da restrição da participação, visto que a maioria das atividades oferecidas da não exige uma escolaridade mínima. Por outro lado, o termo "universidade aberta", ainda pouco difundido na Região Nordeste, pode passar à população a ideia associada à de uma universidade na qual não se ingressa caso não haja escolaridade e aprovação em avaliações no formato tradicional de provas.

Neste sentido, é preciso que as UATIs busquem atrair para si esta população, até então comumente excluída deste tipo de projeto, tendose em vista também que, quanto maior a escolaridade, maior a chance de sobrevivência e de resistência às demandas do dia a dia, e, portanto, torna-se imprescindível que o processo educacional e de aprendizagem se estenda por toda a vida. O planejamento das ações educacionais nesse tipo de instituição deve considerar as necessidades do grupo e individuais, como também respeitar a heterogeneidade $d a$ população, buscando atingir uma parcela mais representativa do todo. ${ }^{7,10}$

Em relação ao estado civil, apresentado na tabela 7, observa-se um importante percentual de mulheres viúvas, tanto no Brasil e em Alagoas, quanto na UATI, embora nesta a proporção tenha sido um pouco menor, o que se justifica por se tratar de indivíduos mais jovens, em boa parte ainda casados. A grande proporção de viúvos acontece com os idosos, em sua maioria mulheres, pelo fato de elas viverem mais que os homens, além de a maioria delas ter-se casado com homens um pouco mais velhos. Quando ocorre o divórcio, os idosos tendem a se casar novamente com mulheres bem mais jovens, diferentemente das idosas. ${ }^{2,10,15,16}$

Em relação à participação nas atividades em comunidade, conforme apresentado nos resultados, na população estudada, observou-se que a maioria dos estudantes da UATI direcionava seu tempo livre às atividades religiosas, de forma semelhante ao encontrado em estudo anterior, ${ }^{2}$ em que a prática de atividades religiosas está entre as mais realizadas pelas idosas da UATI de Pernambuco. Semelhanças entre ambos os estudos também foram verificados em relação à prática de atividade física, relatada por pequena parcela das amostras (11,3\% dos participantes da UATI alagoana e 5,19\% das idosas em Pernambuco).

A maior parcela dos participantes da UATI via no programa uma forma de buscar crescimento pessoal, lazer e distração. Em estudo anterior, de proposta para a implantação de uma Universidade à Terceira Idade em Joinville, verificou-se que, quando questionados sobre o interesse de retornar à escola, 69\% dos idosos entrevistados responderam que queriam retornar os estudos, $\mathrm{e}$ $49 \%$ deles declararam que o maior interesse estava em melhorar o nível cultural. ${ }^{13}$ Neste sentido, por meio dos dados anteriormente apresentados, cabe salientar que as propostas e objetivos das UATIS estão afinadas com os anseios de seu público-alvo, já que trazem como propostas oportunidades de acesso à educação, cultura e lazer, proporcionando a interação social. Por outro lado, proporcionar educação, cultura e lazer a idosos são formas de se alcançar outro objetivo: o de se promover saúde, que apareceu em poucos relatos dos idosos, talvez por desconhecerem esta função, possivelmente pouco divulgada como tal na sociedade. A capacidade de interagir socialmente é fundamental para o idoso, a fim de que ele possa conquistar e manter as redes de apoio social e garantir maior qualidade de vida. ${ }^{18} \mathrm{~A}$ possibilidade de conhecer melhor os mecanismos biológicos, sociais e culturais envolvidos com esta 
faixa etária, propiciado pela UATI, também pode proporcionar ao idoso melhor gerenciamento sobre sua condição de saúde.

A maioria dos participantes deste estudo obteve informações sobre a UATI por meio da televisão, o que mostra a importância deste meio de divulgação para a disseminação das propostas deste programa. A divulgação entre amigos e através dos funcionários da UATI foi, respectivamente, a segunda e terceira maior frequência relatada. Nota-se, portanto, a importância do envolvimento de todos os profissionais da universidade na divulgação desta proposta, assim como o investimento na satisfação do público-alvo, pois foram eles um dos maiores meios de disseminação deste programa. Percebese que poucos selecionaram ter obtido informações através de meios impressos (jornais). Pode-se atribuir esse fato à pouca divulgação através desta ferramenta, sendo importante, assim, intensificar a disseminação do programa através de outros meios de comunicação, já que através dele é possível esclarecer mais a população-alvo e aqueles a ela ligados, sobre este programa.

\section{CONCLUSÃO}

O perfil encontrado não reflete a realidade brasileira, à semelhança do que se encontra em outras UATIs brasileiras, ressaltando a necessidade de que elas, enquanto programas de promoção à saúde, incluam os idosos de classes socioeconômicas e culturais menos favorecidas.

Os dados se limitam a um estado que tem índices sociais perversos e diversos em relação aos demais do próprio Nordeste. Por só haver registros de estudos semelhantes nesta região no Estado de Pernambuco, é evidente a necessidade de se ampliar as pesquisas sobre o tema.

Estudos desta natureza permitem maior fundamentação, tanto aos projetos em andamento, quanto aos planejados, no sentido de se identificar as atividades condizentes com os anseios e realidade dos que frequentam a UATI, visando, porém, a atingir também uma parcela da população não contemplada.

\section{AGRADECIMENTOS}

Ao Prof. Dr. André Falcão Pedrosa Costa e ao Prof. Dr. Guilherme Benjamin Brandão Pitta pela participação efetiva na concretização do Projeto "Universidade Aberta à Terceira Idade" da Universidade Estadual de Ciências da Saúde de Alagoas (UNCISATI). Agradecemos também aos profissionais, professores e estudantes que integram o Programa de Extensão Interdisciplinar Pró-Idoso, dentro do qual a UNCISATI se insere e que participaram desde o planejamento até a efetivação da UNCISATI. 


\section{REFERÊNCIAS}

1. Fundação Instituto de Geografia e Estatística . Síntese de Indicadores Sociais: Uma Análise das Condições de Vida da População Brasileira. Estudos e Pesquisas Informações Demográficas e Sócio-Econômica, 2007 (21) 149-177.

2. Barreto KML, Carvalho EMF, Falcão IV, Lessa FJD, Leite VMM. Perfil sócio-epidemiológico demográfico das mulheres idosas da Universidade Aberta à Terceira Idade no estado de Pernambuco. Rev. Bras. Saude Mater. Infant. [periódico na Internet] 2003 Set [Acesso em 9 Out 2007]; 3(3): 339-354. Disponível em: http:// www.scielo.br/scieloOrg/php/ articleXML.php?pid=S1519$38292006000100004 \&$ lang $=$ en

3. World Health Organization. Envelhecimento ativo: uma política de saúde / World Health Organization; tradução Suzana Gontijo. Brasília: Organização Pan-Americana da Saúde; 2005.60.

4. Organização Mundial de Saúde. Declaração Elaborada pelo Grupo de Trabalho da Qualidade de Vida da OMS: glossário de Promoção de Saúde da OMS de 1998.in: OMS/ HPR/HEP/98.i Genebra: Organização Mundial da Saúde, 1994.

5. Pesquisa Nacional por Amostra de Domicílios do IBGE. Um diagnóstico sócio-econômico do Estado de Alagoas a partir de uma leitura dos dados da Pesquisa Nacional por Amostra de Domicílios do IBGE. Instituto de estudos do trabalho e sociedade; 2005.

6. Gonçalves LHT, Alvarez AM, Sena ELS, Santana LWS, Vicente FR. Perfil da família cuidadora de idoso doente fragilizado do contexto sociocultural de Florianópolis. Texto $\&$ contexto enferm [periódico na internet]. 2006 Out/Dez [Acesso em 10 Out 2007 ]; 15(4):570577. Disponível em: http://www.scielo.br/ scielo.php?script $=$ sci_arttext\&pid $=$ S010407072007000100026\&lng $=\mathrm{pt \& nrm}=$ iso\&tlng $=\mathrm{pt}$

7. Veras RP, Caldas CP. Promovendo a saúde e a cidadania do idoso: o movimento das universidades da terceira idade. Ciênc. saúde coletiva [periódico na Internet]. 2004 Jun [Acesso em 16 Out 2007]; 9(2): 423-432. Disponível em: http://www.scielo.br/ scielo.php?pid $=$ S1413$81232004000200018 \&$ script $=$ sci_abstract\&tlng $=$ pt

8. Organização das Nações Unidas. Plano internacional de ação sobre o envelhecimento. Asemblea Mundial sobre El envejecimiento 2. Madrid; 2002.
9. Teixeira-Salmela LF, Magalhães LC, Souza AC, Lima MC, Lima RCM, Goulart F. Adaptação do Perfil de Saúde de Nottingham: um instrumento simples de avaliação da qualidade de vida. Cad Saúde Pública [periódico na Internet]. 2004 Ago [Acesso em 09 Out ]; 20(4): 905-14. Disponível em: http://www.lava.med.br/ MESTRADO/VASCULAR/2005/

Artigos_Revista/

Modulo_XXXIV_Qualidade_de_vida/ Adaptacao_do_Perfil_de_Saude_de_Nottingham.pdf.

10. Anderson MIP, Assis M, Pacheco LC, Silva EAS, Menezes IS, Duarte T, Storino F, Motta L. Saúde e qualidade de vida na terceira idade. Textos envelhecimento [periódico na internet]. 1998 Nov [Acesso em 12 Out]; 1(1): 23-43. Disponível em: http://bases.bireme.br/cgi-bin/ wxislind.exe/iah/online/?IsisScript $=$ iah/ iah. $x$ is\&src $=$ google\&base $=$ LILACS\&lang $=$ $p \&$ nextAction $=$ lnk\&exprSearch $=291151$ \&indexSearch $=\mathbb{D}$

11. Assis M, Hartz ZMA, Valla VV. Programas de promoção da saúde do idoso: uma revisão da literatura científica no período de 1990 a 2002. Ciênc. saúde coletiva [periódico na Internet]. 2004 Set [acesso em 30 Out ]; 9 (3): 557-581. Disponível em: http://www.scielosp.org/ scielo.php?script $=$ sci_arttext\&pid $=$ S141381232004000300010\&lng $=\mathrm{pt}$

12. Leite VMM, Carvalho EMF, Barreto KML, Falcão IV. Depressão e envelhecimento: estudo nos participantes do Programa Universidade Aberta à Terceira Idade. Rev. Bras. Saude Mater. Infant. [periódico na Internet]. $2006 \mathrm{Mar}$ [Acesso em 09 Out 2007]; 6(1):31-38. Disponível em: http://www.scielo.br/ scielo.php?script $=$ sci_arttext\&pid $=$ S1519$38292006000100004 \& \ln \mathrm{g}=\mathrm{pt} \& \mathrm{nrm}=$ iso\&tlng $=\mathrm{PT}$

13. Machado OG. Proposta de Implantação de Universidade Aberta para a Terceira Idade em Joinville. Tese [ doutorado] - Universidade Federal de Santa Catarina; 2003.

14. Fenalti RCS, Schwartz GM. Universidade aberta à terceira idade e a perspectiva de ressignificação do lazer. Rev paul Educ fís [periódico na internet] $2003 \mathrm{jul} / \mathrm{Dez}$ [Acesso 15 Out 2007 ]; 17(2):131-141. Disponível em: http:// www.usp.br/eef/rpef/v17n22003/v17n2p131.pdf

15. Venturi G . Idosos no Brasil: vivências, desafios e expectativas na terceira idade. Fundação Perseu Abramo; 2007.

16. Santos SR, Santos IBC, Fernandes MGM, Henriques ME, Romero M. Qualidade de vida do idoso na comunidade: aplicação da Escala de 
Flanagan. Rev Latino-Am

Enfermagem [periódico na Internet]. $2002 \mathrm{Dez}$

[Acesso em 09 Out 2007 ]; 10(6): 757-764.

Disponível em: http://www.scielo.br/

scielo.php?script $=$ sci arttext\&pid $=$ S0104-

$11692002000600002 \& \operatorname{lng}=\& \mathrm{nrm}=\mathrm{iso} \& \mathrm{t} \operatorname{lng}=$

17. Tavares EL, Anjos LA. Perfil antropométrico da população idosa brasileira :resultados da Pesquisa Nacional sobre Saúde e Nutrição. Cad Saúde Pública [Periódico Internet], 1999 out-dez. [Acesso em 2007 out 27] 15(4):759-768.

Disponível em: http://www.scielosp.org/ scielo.php?script $=$ sci_arttext\&pid $=\mathrm{S} 0102$ 311X1999000400010

18. Carneiro RS, Falcone EMO. Um estudo das capacidades e deficiências em habilidades sociais na terceira idade. Psicol. estud [periódico na internet]. $2004 \mathrm{jan} / \mathrm{abr}$ [Acesso em 13 Out 2007 ]; 9(1):119-126. Disponível em: http://www.scielo.br/ scielo.php?pid = S1413-

$73722004000100015 \&$ script $=$ sci_arttext
19. Ramos LR. Fatores determinantes do envelhecimento saudável em idosos residentes em centro urbano: projeto Epidoso, São Paulo. Cad Saúde Pública [periódico na Internet]. 2003 Jun [Acesso em 09 Out 2007 ]; 19(3): 793-797. Disponível em: http://www.scielosp.org/ scielo.php?script $=$ sci_abstract\&pid $=$ S0102$311 \mathrm{X} 2003000300011 \& \mathrm{nrm}=$ iso\&tlng $=\mathrm{pt}$

20. Vecchia RD, Ruiz T, Bocchi SCM, Corrente JE. Qualidade de vida na terceira idade: um conceito subjetivo. Rev bras epidemiol [periódico na Internet]. 2005 Set [Acesso em 09 Out 2007 ]; 8(3): 246-252. Disponível em: http:// www.scielo.br/pdf/rbepid/v8n3/06.pdf

21. Pereira RJ , Cotta RMM . Contribuição dos domínios físico, social, psicológico e ambiental para a qualidade de vida global de idosos. Ver Psiquiatr Rio Gd. Sul [periódico na internet] 2006 jan/abr [Acesso em 22 Out 2007 ];28(1).

Disponível em: http://www.revistapsiqrs.org.br/ administracao/arquivos/

contribuicao_dominios_fisicos_28_01_06.pdf 